\title{
An Infrequent Case of Extra Abdominal Fibromatosis
}

\author{
M. Sabari Girieasen", Naveenkumar Viswanathan, S. Nedunchezhian, Kannan Ross
}

Institute of General Surgery, Madras Medical College, Chennai, Tamilnadu, India

DOI: $\underline{10.36347 / \mathrm{sasjs} .2020 . \mathrm{v} 06 \mathrm{i} 02.011}$

| Received: 14.02.2020 | Accepted: 21.02.2020 | Published: 22.02.2020

*Corresponding author: Dr. M. Sabari Girieasen

Abstract

Case Report

Extra-abdominal fibromatosis, also known as a desmoid tumor, is a rarely observed benign lesion, characterized as a non-metastatic lesion with local invasiveness. These tumors are due to proliferation of the fibroblast and common in females. A 40 year old male developed a swelling in the right thigh post traumatic and it progressively increased to attain present size. On examination, a single, solid, oval, firm, smooth surfaced, ill-defined swelling of size $10 \mathrm{x} 8 \mathrm{~cm}$ is noted in the proximal thigh. MRI showed a space occupying lesion in the intramuscular region between rectus femoris and tensor fascia lata. Core needle biopsy came as possibility of spindle cell neoplasm. Radical surgical excision showed a poorly circumscribed lesion with clear margins, and IHC study showed beta catenin and CD31 positivity. The diagnosis was in favor of fibromatosis. Patient was discharged and there was no recurrence. This patient received the optimal treatment modality and he is under careful surveillance for recurrence.

Keywords: Extra abdominal fibromatosis, desmoid tumor, thigh, surgery.

Copyright @ 2020: This is an open-access article distributed under the terms of the Creative Commons Attribution license which permits unrestricted use, distribution, and reproduction in any medium for non-commercial use (NonCommercial, or CC-BY-NC) provided the original author and source are credited.

\section{INTRODUCTION}

Extra-abdominal fibromatosis, also known as a desmoid tumor, is a rarely observed lesion of benign biological significance, characterized as a non-metastatic lesion with local invasiveness. Extra-abdominal fibromatosis represents $<0.03 \%$ of all neoplasms, with an annual incidence of 2-4 cases/1,000,000 individuals, worldwide. Extra-abdominal fibromatosis occurs more often in females and has a higher incidence between puberty and the fourth decade of life [1].

The most common locations are the shoulder and upper limb (33\%), gluteus and lower extremities $(30 \%)$, chest wall and spine (17\%), and head and neck $(10 \%)$. The desmoid tumor may be primary or secondary to trauma, including surgery, or hormonal stimuli. Magnetic resonance imaging (MRI) is the gold standard technique for diagnosis [2].

The present study reports a case of 40 year with swelling in the right thigh diagnosed as extraabdominal fibromatosis. It is very uncommon in the thigh and the optimal treatment is not standardized.

\section{CASE REPORT}

A 40 year old male who is a laborer by occupation with no comorbidities presented with complaints of swelling in the right thigh for one year.
Initially he noticed a swelling which was small in size and gradually it increased to attain the present size. He had a history of trauma one year back. No history of fever, pain, loss of weight and appetite, difficulty in walking and restriction of movements. No other swelling elsewhere in the body and no addictive habits.

On admission, vitals were stable. On examination, a single solid oval swelling of size $10 \times 8$ $\mathrm{cm}$ noted in the upper $1 / 3^{\text {rd }}$ of the anterior aspect of right thigh eextending $8 \mathrm{~cm}$ below ASIS and $20 \mathrm{~cm}$ above the patella (Figure 1). There was no local warmth and tenderness. Swelling is smooth surfaced, margins indistinct, firm in consistency, non-fluctuant and nontrans illuminant. Skin was pinch able and swelling reduces on extending the knee. Swelling had a dull note on percussion.

Routine blood investigations were normal and viral markers were negative. FNAC came as a possibility of spindle cell neoplasm. MRI was taken which showed poorly circumscribed capsulated space occupying lesion in the proximal thigh in the intramuscular plane in between rectus femoris and tensor fascia lata (Figure 2). Mass was free from neurovascular bundle. Core needle biopsy was done and it was suggestive of spindle cell neoplasm. 


\section{RESULTS}

Wide local excision was planned. Skin incision was made and wound deepened in layers. Deep fascia was opened (Figure 3) and sartorius muscle was retracted. Tumor was found to be in rectus femoris and preceded with radical excision of the tumor with $2 \mathrm{~cm}$ clearance (Figure 4). Specimen was sent for histopathological examination (Figure 5). Wound was closed and DT was placed.

Biopsy came as poorly circumscribed lesion with clear margins and features were suggestive of spindle cell neoplasm. Immunohistochemistry showed beta catenin, vimentin and CD 31 positivity with S100 negativity. It goes in favour of fibromatosis. Patient is still in follow up and no recurrence was noted.

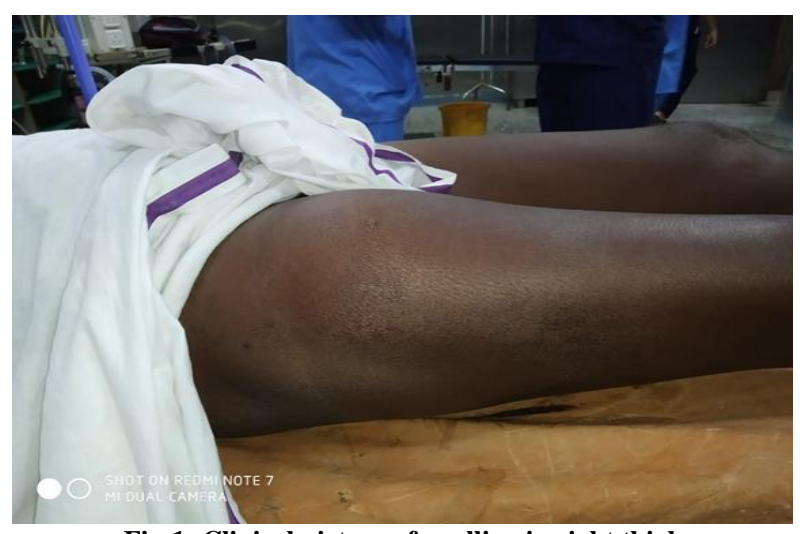

Fig-1: Clinical picture of swelling in right thigh

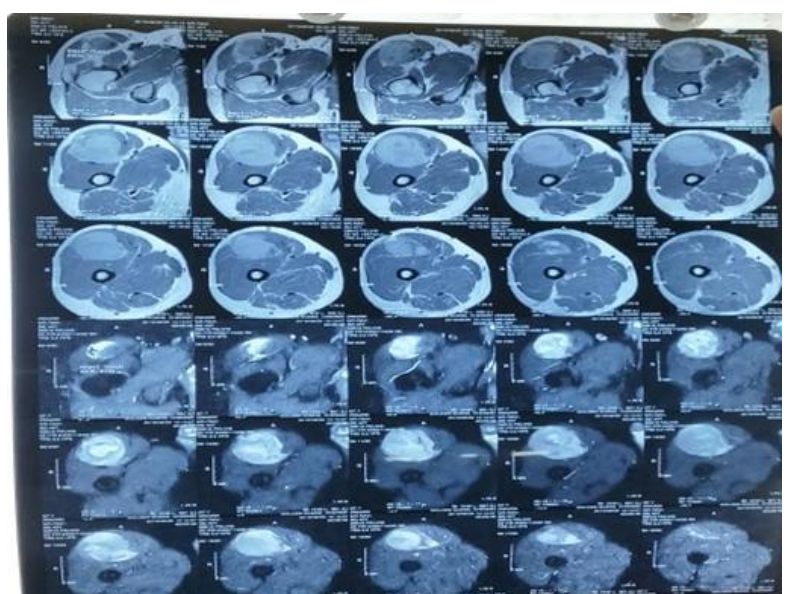

Fig-2: MRI of right thigh showing a mass in the intramuscular space

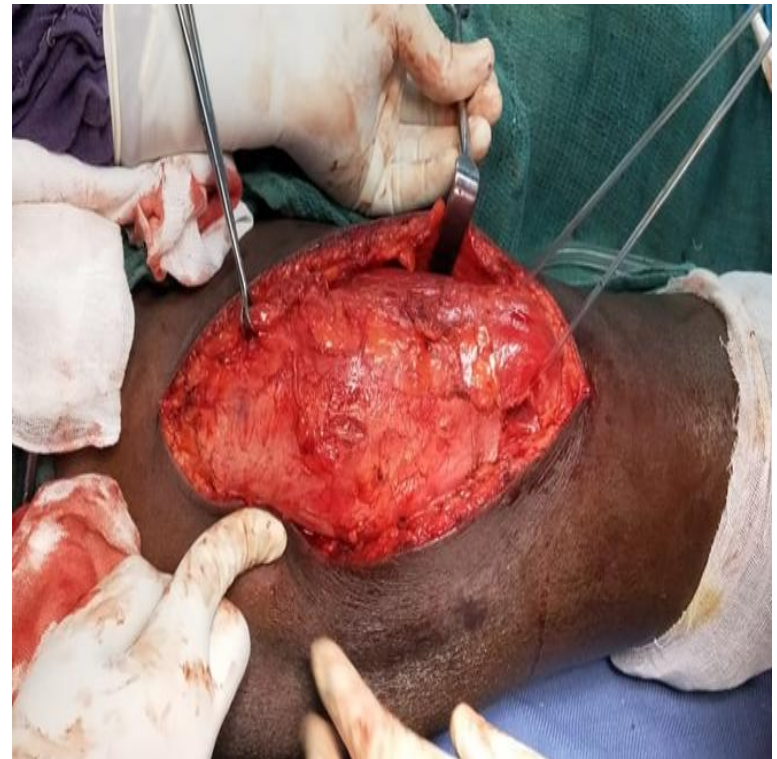

Fig-3: Intro op picture

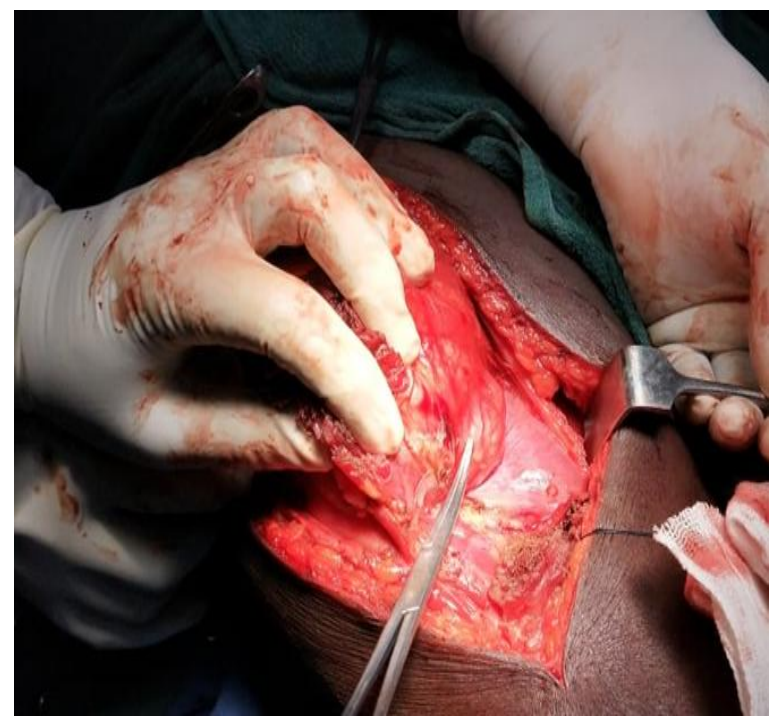

Fig-4: Wide local excision

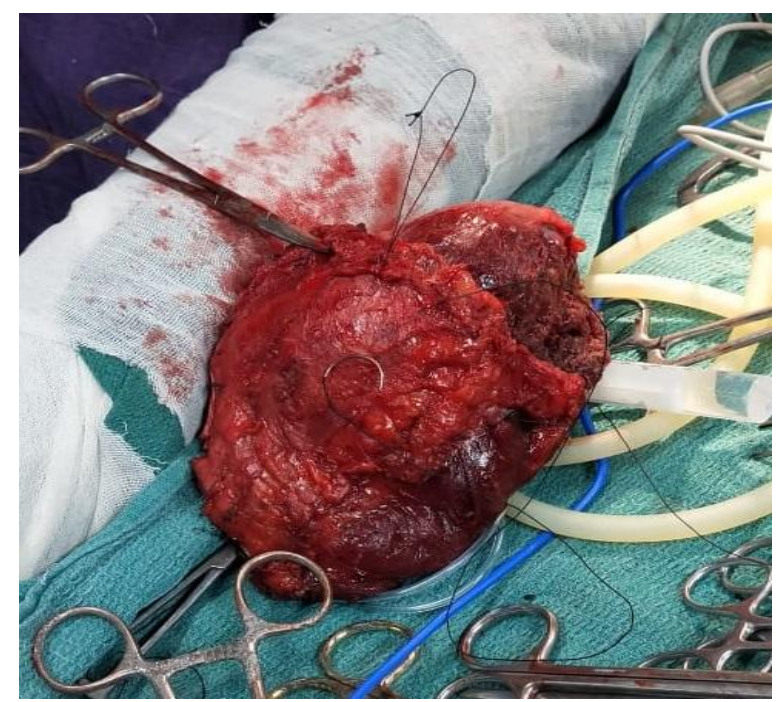

Fig-5: Wide local excision specimen (Tumor was removed with 2 cm clearance) 


\section{DISCUSSION}

Fibromatosis are very rare soft tissue tumors with proliferation of fibroblast and most commonly involving the abdominal wall occurring in the rectus and abdominal scar. It has highly infiltrative with negligible metastatic potential and considered a benign entity. They can arise from any skeletal muscle without skin involvement. They have a high chance of local recurrence.

Possible risk factors for the development of desmoid tumors include female sex, or a previous history of surgery, trauma, or pregnancy. Genetic susceptibility is believed to play a role in the development of the disease, but genetic risk factors have not been identified. Kaygin reported a similar case of swelling in the popliteal fossa which proved to be fibromatosis rather than a vascular tumor [3].

Paramanandhan et al. reported a case of 30 year old woman who had fibromatosis of foot and pulmonary metastasis. In view of metastasis, the patient was managed with palliative chemotherapy. Yet the patient succumbed to disease. Though fibromatosis is considered a benign entity, it can metastasis and can transform to fibrosarcoma [4].

Tiwari et al. reported a similar case of fibromatosis in thigh and wide local excision was done for that patient [5]. Bouffier advocated wide excision in 1899, and it has remained the treatment of choice. Adjunctive chemotherapy and endocrine therapy have been studied, but the results are unclear. Radiotherapy in addition to surgical excision has been shown to be helpful in the management of these tumors [6].

This case is one of rare presentation of fibromatosis in thigh while the most common occurrence is in abdomen. Patient is a male and there was an association with trauma for this patient. Radical resection with free margins remains the treatment of choice. As biopsy had negative margins, chemo and radiotherapy was not given for this patient.

\section{CONCLUSION}

This patient had wide local excision with negative margin for tumor which still remains the optimal treatment modality for extra-abdominal fibromatosis. Patient is under careful surveillance as recurrence is common after excision.

\section{DECLARATIONS}

Funding: The authors have not declared a specific grant for this research from funding agency in the public, commercial or not for profit sectors.

Conflict of interest: none declared

Patient consent for publication: Obtained.

\section{REFERENCES}

1. Escobar C, Munker R, Thoma JO, Li BD and Burton GV: Update on desmoid tumors. Ann Oncol. 23: 562-569, 2012.

2. Fornaro R, Caratto E, Caratto M, Salerno A, Sarocchi F, Minetti G, Frascio M, Murialdo R, Taviani M. Extra-abdominal fibromatosis: Clinical and therapeutic considerations based on an illustrative case. Oncology letters. 2015 Nov 1;10(5):3103-6.

3. Mehmet Ali Kaygin. Case reports in Vascular Medicine; 2011.

4. Paramanandhan M, Kapoor A, Singhal MK, Kumar R, Kumar HS. Pulmonary metastasis from desmoid tumor of the foot.Clin Cancer Investig $\mathrm{J}$. 2015;4:658-661

5. Tiwari A, Topno M, Karim T, Sharma V. A Rare Case of Desmoid Tumor of Thigh. Indian Journal of Surgery. 2010 Oct 1;72(5):409-11.

6. MacGill AA, Milione VR, Sullivan LG. Extraabdominal desmoid fibromatosis in the foot: a case study. Journal of the American Podiatric Medical Association. 2011 Jan;101(1):70-4. 